\title{
Research Article \\ Economic Design of Acceptance Sampling Plans in a Two-Stage Supply Chain
}

\author{
Lie-Fern Hsu' ${ }^{1}$ and Jia-Tzer $\mathrm{Hsu}^{2}$ \\ ${ }^{1}$ Department of Management, Zicklin School of Business, Baruch College, The City University of New York, \\ One Bernard Baruch Way, P.O. Box B9-240, New York, NY 10010, USA \\ ${ }^{2}$ Department of Computer Science and Information Engineering, Hungkuang University, Shalu, \\ Taichung 43302, Taiwan
}

Correspondence should be addressed to Lie-Fern Hsu, lie-fern.hsu@baruch.cuny.edu

Received 2 August 2011; Revised 13 January 2012; Accepted 19 January 2012

Academic Editor: Henry Schellhorn

Copyright (C 2012 L.-F. Hsu and J.-T. Hsu. This is an open access article distributed under the Creative Commons Attribution License, which permits unrestricted use, distribution, and reproduction in any medium, provided the original work is properly cited.

\begin{abstract}
Supply Chain Management, which is concerned with material and information flows between facilities and the final customers, has been considered the most popular operations strategy for improving organizational competitiveness nowadays. With the advanced development of computer technology, it is getting easier to derive an acceptance sampling plan satisfying both the producer's and consumer's quality and risk requirements. However, all the available $Q C$ tables and computer software determine the sampling plan on a noneconomic basis. In this paper, we design an economic model to determine the optimal sampling plan in a two-stage supply chain that minimizes the producer's and the consumer's total quality cost while satisfying both the producer's and consumer's quality and risk requirements. Numerical examples show that the optimal sampling plan is quite sensitive to the producer's product quality. The product's inspection, internal failure, and postsale failure costs also have an effect on the optimal sampling plan.
\end{abstract}

\section{Introduction}

Supply Chain Management focuses on the material and information flows between facilities and their final customer, and has been considered the most popular operations strategy for improving organizational competitiveness in the 21st century [1]. Recently, due to the pressure to lower manufacturing and service costs and to deliver high-quality products to market quickly, North American companies have become increasingly attracted to outsourcing and off-shoring, which is the usage of overseas workers to produce components, entire products, and services. Many companies have contracted with suppliers in lower-cost countries in order to gain access to a large pool of workers at a mere fraction of the cost 
of domestic facilities. For this reason, India and China are becoming major players in offshoring, especially in the areas of manufacturing and service.

Recent product recall scandals have revealed that the benefits of outsourcing and offshoring also come with its disadvantages - in this case, the threat of quality risks in the supply chain. Some examples of recent product recalls include Toyota's sticking accelerator pedal recall and floor mat recall (e.g., vehicles involved in the sticking accelerator pedal recall include: 2007-2010 Camry, 2009 Camry Hybrid, 2009-2010 Corolla, 2009-2010 RAV4, 2010 Highlander; vehicles involved in the floor mat recall include: 2007-2010 Camry, 2009-2010 Corolla, 2008-2010 Highlander) and China's recent toys, pet food, and melamine milk recalls.

Cao and Zhang [2] showed that firms have been attempting to achieve greater collaborative advantages with their supply chain partners in the past few decades, and that supply chain collaborative advantages have a bottom-line influence on firm performance. In addition, Foster Jr. [3] established that the increasing importance given to supply chain management has resulted in the rethinking of models, constructs, and frameworks for quality management that have been developed for operations management. Although research in quality management has previously focused on an internal versus external view of quality, where the internal view focused on process and the external on the customers, companies must now merge these views as they adopt the systems approach implicitly in supply chain management, in order to internalize upstream and downstream processes with their own. Thus, Foster Jr. [3] defined Supply Chain Quality Management (SCQM) as a system-based approach to performance improvement that leverages opportunities created by upstream and downstream linkages with suppliers and customers.

The purpose of this paper is to design an economic model to determine the optimal sampling plan in a two-stage supply chain that minimizes the producer's and the consumer's total quality cost while satisfying both the producer's and the consumer's quality and risk requirements. The model can be applied to any two-stage supply chain including a vendor and a buyer, where a vendor deliver a batch of product to the buyer, and the buyer decides whether to accept or reject the entire lot based on the quality of the sample selected from the lot. Acceptance sampling is often used to monitor the quality of raw material, purchased parts, and finished products when product testing is destructive, time-consuming, or expensive. An acceptance plan is the overall scheme for either accepting or rejecting a lot based on information gained from samples. The acceptance plan identifies both the size and type of samples and criteria to be used to either accept or reject the lot. Samples may be either single, double, multiple, or sequential.

Single sampling plans are simple to use. However, if the incoming quality level is particularly good or particularly poor, a double, multiple, or sequential sampling plan will reach an acceptance or a rejection decision sooner and, therefore, reduce the average sample number. Moreover, if a single sampling plan is applied, very often the producer is at a "psychological" disadvantage, since no second chance is given for the rejected lots. In such situations, taking a second sample is preferable.

In a single sampling plan, one sample of items is selected at random from a lot and the disposition of the lot is determined from the resulting information. These plans are usually denoted as $(n, c)$ plans for a sample size $n$, where the lot is rejected if there are more than $c$ defectives. These are the most common (and easiest) plans to use, although not the most efficient in terms of the average number of samples needed.

In a double sampling plan, after the first sample is tested, there are three possibilities:

(1) accept the lot, 
(2) reject the lot,

(3) take a second sample.

If the outcome is (3), a second sample is taken, and the procedure is to combine the results of both samples and make a final decision based on that information.

A multiple sampling plan is an extension of the double sampling plans where more than two samples are needed to reach a conclusion. The advantage of multiple sampling is smaller sample sizes.

A sequential sampling plan is the ultimate extension of multiple sampling where items are selected from a lot one at a time and after inspection of each item a decision is made to accept or reject the lot or select another unit.

One of the most common ways to set the sampling plan parameters is to use what are often referred to as QC tables. The two most common sets of these tables are as follows.

\section{(1) ANIS/ASQ Z1.4 and Z1.9-2008 [see [4]]}

Using the sample size code letter (which is determined by the shipping lot size and the inspection level), the sampling plan can be read off instantly for a specified acceptable quality level (AQL). The AQL is a percent defective that is the base line requirement for the quality of the producer's product. The producer would like to design a sampling plan such that there is a high probability of accepting a lot that has a defect level less than or equal to the AQL. It provides tightened, normal, and reduced plans to be applied for attributes inspection for percent nonconforming or nonconformities per 100 units $[4,5]$. The producer's risk (Type I error) is the probability, for a given sampling plan, of rejecting a lot that has a defect level equal to the AQL. The producer suffers when this occurs, because a lot with acceptable quality is rejected. The symbol $\alpha$ is commonly used for the producer's risk and the typical value for $\alpha$ is 0.05 .

\section{(2) Dodge-Romig Tables}

These attribute acceptance plans set the parameters while assuming the rejected lots are 100 percent inspected and defectives are replaced with nondefectives. Users must specify values for consumer's risk $(\beta)$, the approximate actual percent defectives, the lot size $(N)$, and the lot tolerance percent defective (LTPD) [6]. The LTPD is a designated high defect level that would be unacceptable to the consumer. The consumer would like the sampling plan to have a low probability of accepting a lot with a defect level as high as the LTPD. The consumer's risk (Type II error) is the probability, for a given sampling plan, of accepting a lot with a defect level equal to the LTPD. The consumer suffers when this occurs, because a lot with unacceptable quality is accepted. The symbol $\beta$ is commonly used for the Type II error and the typical value for $\beta$ is 0.10 .

Some computer software packages are available now to find the acceptance sampling plans that satisfy the company's quality and risk requirements. For example, Sampling Plan Analyzer 2.0 [7] is a shareware software package for evaluating and selecting acceptance sampling plans. Users can take an existing sampling plan and use the software to evaluate it including calculating and displaying OC (Operating Characteristic) curves (the OC curve plots $p_{a}$, the probability of accepting the lot $(Y$-axis) versus $p$, the lot fraction, or percent defectives ( $X$-axis); the OC curve is a graph of the performance of an acceptance sampling plan, it shows how well an acceptance plan discriminates between good and bad lots). Users 
can also specify the desired protection and the program will generate a list of sampling plans that might be used.

A plot of the Average Outgoing Quality (AOQ, $Y$-axis) versus the incoming lot $p$ (Xaxis) will start at 0 for $p=0$ and return to 0 for $p=1$ (where every lot is $100 \%$ inspected and rectified). The AOQ curve shows that as $p$, the lot fraction or percent defectives, increases, the AOQ initially deteriorates and then improves. The improvement in quality occurs because as the acceptance plan rejects lots, the rejected lots are 100 percent inspected and defectives are either replaced with nondefectives or removed. In between, it will rise to a maximum. This maximum, which is the worst possible long-term AOQ, is called the AOQL.

Acceptance Sampling for Attribute TP105 [8] develops sampling plans for attribute data based on the binomial and the Poisson distributions. The metric used for the OC curve can be either the fraction defective, as in the binomial case of go/no-go data, or counts, as in the Poisson case of defect count.

All the previous QC tables or computer software $[7,8]$ determine the sampling plans on a non-economic basis to satisfy the quality and risk requirements of the producer, the consumer, or both parties. Motivated by the case of a Greek company, which uses the Greek equivalent to the ISO 2859 [9] for the quality control of its incoming raw materials, Nikolaidis and Nenes [10] evaluated the single-sampling plans recommended by the international standard ISO 2859 from an economic point of view. Their evaluation shows that the use of the ISO 2859 rarely leads to satisfactory economic results. Wetherill and Chiu [11] reviewed some major principles of acceptance schemes with emphasis on the economic aspect. Their research indicated that the major approaches for designing an economic acceptance sampling plan include the following.

(1) The Bayesian approach. This approach assesses the costs and losses involved in operating a sampling plan and tries to minimize the total costs. The expected cost per batch includes the cost of sampling and the loss due to wrong decisions, which is a function of the process quality $p$. The optimal single sampling plan $(n, c)$ is obtained by minimizing the expected cost per batch with respect to these two variables.

(2) The Minimax Approach. This approach also aims at minimizing costs but without assuming a knowledge of the process quality $p$. Thus the average cost per batch $C(p)$ is a function of $p$. For any given sampling plan, $C(p)$ will go through a maximum as $p$ runs from 0 to 1 . The minimax principle chooses the plan that minimizes this maximum.

(3) Semieconomic Approach. Here a point on the OC curve is specified. The fixed point on the OC curve can be the producer's risk point, the consumer's risk point, or the point of indifference quality. The fixed point determines a relationship between $c$ and $n$. The plan that minimizes the average amount of inspection at the process average quality is chosen.

Tagaras [12] developed an economic model to assist in the selection of minimum cost acceptance sampling plans by variables. The quadratic Taguchi loss function is adopted to model the cost of accepting items with quality characteristics deviating from the target value. Ferrell Jr. and Chhoker [13] presented a sequence of models that addressed $100 \%$ inspection and single sampling with and without inspector error when a Taguchi-like loss function is used to describe the cost associated with any deviation between the actual value of a product's quality characteristics and its target value. González and Palomo [14] developed 
a Bayesian acceptance sampling plan for a lot consisting of $N$ units, when the number of defects in a unit can be described by a Poisson distribution with parameter $\lambda$, and the prior distribution of $\lambda$ takes the form of a gamma or noninformative function. In the acceptancesampling plan to be constructed, a sample of size $n$ is taken from a lot of size $N$ and all units in the sample are inspected. If the number of defects found in the sample is above a specified value $c$, the lot is rejected. If the number of defects is at or below $c$, the lot is accepted and sent to the next stage without further inspection. The sampling plans are obtained following an economic criterion: minimize the expected total cost of quality. Note that none of the research available in the literature focusing on the economic design of acceptance sampling plans has integrated the producer's and the consumer's risk requirements into the design of the model.

In this paper, we consider a two-stage supply chain. For example, one of the major agriculture export products from Taiwan is the orchid. According to the Statistics of the Agriculture and Food Agency of the Taiwanese government, the export value of the seedlings of Phalaenopsis (Butterfly Orchid) to all countries was 13,525,800 US dollars for the year of 2010 , among which $\$ 5,497,900$ 's worth was to the USA and $\$ 1,971,500$ 's worth was to the Netherlands. In the USA, once the seedlings of Phalaenopsis arrive at the seaports (California or Florida), they are inspected (100 percent inspection for new suppliers and sampling inspections for old suppliers). The defective products are either scrapped at the seaport or returned to Taiwan. In the Netherlands, the defective products are sold at a reduced price. We will develop the optimal sampling plan based on an economic viewpoint. This paper is organized as follows. Section 2 formulates the optimization problem for the economic design of acceptance sampling plan. Section 3 provides numerical examples to illustrate the effects of quality and costs on the optimal sampling plan. Finally, Section 4 concludes this paper with a brief summary of the main results.

\section{Economic Design of Acceptance Sampling Plan}

Figure 1 illustrates how a single-sampling plan for attributes operates.

Let $p_{a}(p)$ denote the probability of accepting the lot given that the lot quality is $p$ and let ATI denote the average total inspection items. The single sampling plan has the following performance measurements [15]:

$$
\begin{gathered}
p_{a}(p)=\sum_{X=0}^{C}\left(\begin{array}{l}
n \\
x
\end{array}\right) p^{X}(1-p)^{n-X} \\
\mathrm{AOQ}=\frac{p p_{a}(p)(N-n)}{N}
\end{gathered}
$$

if defective items are replaced with good ones and

$$
\mathrm{AOQ}=\frac{p p_{a}(p)(N-n)}{N-\mathrm{np}-\left(1-p_{a}(p)\right) p(N-n)}
$$




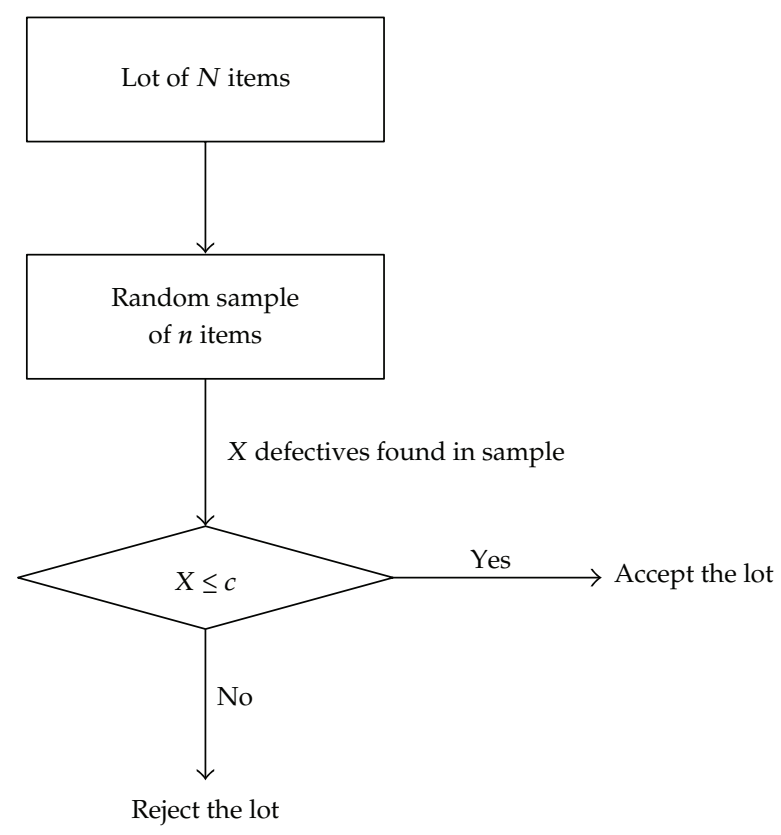

Figure 1: $n$ is the number of items to be sampled; $c$ is the prespecified acceptable number of defectives.

if defective items are removed but not replaced, and

$$
\mathrm{ATI}=n+\left(1-p_{a}(p)\right)(N-n) .
$$

Let $D_{d}$ denote the defective items detected; and let $D_{n}$ denote the defective items not detected, then we have

$$
\begin{gathered}
D_{d}=\mathrm{np}+\left(1-p_{a}(p)\right)(N-n) p, \\
D_{n}=p_{a}(p)(N-n) p .
\end{gathered}
$$

Note that if the inspection is $100 \%$ reliable, for the sampled $n$ items, the expected defective items np will be detected for sure. If the lot is rejected (with probability $1-p_{a}(p)$ ), it will be $100 \%$ inspected and the remaining $(N-n) p$ defective items will be detected. On the other hand, if the lot is accepted (with probability $p_{a}(p)$ ), the $(N-n) p$ defective items will not be detected.

To derive the total quality cost per lot for a given sampling plan, we define the following cost parameters:

$C_{i}$ : Inspection cost per item.

$C_{f}$ : Internal failure cost; that is, the cost of rework, repair, or replacement for a defective item which is not released to the market as a finished product or not released to production as an incoming raw material.

$C_{o}$ : The cost of an outgoing defective item (i.e., the postsale failure cost, see Hsu and Tapiero [16]). For a finished product, this is the cost of replacement and loss of good will for 
Table 1: Single sampling plans satisfying AQL $=0.02, \mathrm{LTPD}=0.07, \alpha=0.05, \beta=0.1$, with $n \leq 205$.

\begin{tabular}{|c|c|c|c|c|c|c|c|c|c|}
\hline TC & $n$ & $c$ & AOQ & ATI & $D_{d}$ & $D_{n}$ & $1-P_{a}(\mathrm{AQL})$ & $P_{a}($ LTPD $)$ & $P_{a}(p)$ \\
\hline 532.64 & 131 & 5 & 0.0208 & 306.10 & 9.18 & 20.82 & 0.0487 & 0.0974 & 0.7985 \\
\hline 518.11 & 149 & 6 & 0.0214 & 286.98 & 8.61 & 21.39 & 0.0310 & 0.0970 & 0.8379 \\
\hline 521.23 & 150 & 6 & 0.0213 & 291.10 & 8.73 & 21.27 & 0.0320 & 0.0934 & 0.8340 \\
\hline 524.38 & 151 & 6 & 0.0211 & 295.24 & 8.86 & 21.14 & 0.0330 & 0.0900 & 0.8301 \\
\hline 527.54 & 152 & 6 & 0.0210 & 299.40 & 8.98 & 21.02 & 0.0340 & 0.0867 & 0.8262 \\
\hline 530.73 & 153 & 6 & 0.0209 & 303.59 & 9.11 & 20.89 & 0.0350 & 0.0835 & 0.8222 \\
\hline 533.93 & 154 & 6 & 0.0208 & 307.80 & 9.23 & 20.77 & 0.0361 & 0.0804 & 0.8182 \\
\hline 537.15 & 155 & 6 & 0.0206 & 312.04 & 9.36 & 20.64 & 0.0372 & 0.0775 & 0.8142 \\
\hline 540.39 & 156 & 6 & 0.0205 & 316.30 & 9.49 & 20.51 & 0.0383 & 0.0746 & 0.8101 \\
\hline 543.64 & 157 & 6 & 0.0204 & 320.58 & 9.62 & 20.38 & 0.0394 & 0.0718 & 0.8060 \\
\hline 546.91 & 158 & 6 & 0.0203 & 324.89 & 9.75 & 20.25 & 0.0406 & 0.0690 & 0.8018 \\
\hline 550.20 & 159 & 6 & 0.0201 & 329.21 & 9.88 & 20.12 & 0.0417 & 0.0664 & 0.7976 \\
\hline 553.50 & 160 & 6 & 0.0200 & 333.55 & 10.01 & 19.99 & 0.0429 & 0.0639 & 0.7934 \\
\hline 556.82 & 161 & 6 & 0.0199 & 337.92 & 10.14 & 19.86 & 0.0441 & 0.0615 & 0.7891 \\
\hline 560.15 & 162 & 6 & 0.0197 & 342.30 & 10.27 & 19.73 & 0.0453 & 0.0591 & 0.7848 \\
\hline 563.49 & 163 & 6 & 0.0196 & 346.70 & 10.40 & 19.60 & 0.0466 & 0.0568 & 0.7805 \\
\hline 566.85 & 164 & 6 & 0.0195 & 351.11 & 10.53 & 19.47 & 0.0479 & 0.0546 & 0.7762 \\
\hline 570.22 & 165 & 6 & 0.0193 & 355.55 & 10.67 & 19.33 & 0.0492 & 0.0525 & 0.7718 \\
\hline 507.35 & 166 & 7 & 0.0218 & 272.82 & 8.18 & 21.82 & 0.0192 & 0.0991 & 0.8719 \\
\hline 509.99 & 167 & 7 & 0.0217 & 276.31 & 8.29 & 21.71 & 0.0199 & 0.0957 & 0.8688 \\
\hline 512.66 & 168 & 7 & 0.0216 & 279.82 & 8.39 & 21.61 & 0.0205 & 0.0924 & 0.8656 \\
\hline 515.35 & 169 & 7 & 0.0215 & 283.35 & 8.50 & 21.50 & 0.0212 & 0.0892 & 0.8624 \\
\hline 518.05 & 170 & 7 & 0.0214 & 286.91 & 8.61 & 21.39 & 0.0218 & 0.0861 & 0.8591 \\
\hline 520.78 & 171 & 7 & 0.0213 & 290.50 & 8.72 & 21.28 & 0.0225 & 0.0830 & 0.8559 \\
\hline 523.52 & 172 & 7 & 0.0212 & 294.11 & 8.82 & 21.18 & 0.0232 & 0.0801 & 0.8525 \\
\hline 526.29 & 173 & 7 & 0.0211 & 297.75 & 8.93 & 21.07 & 0.0239 & 0.0773 & 0.8492 \\
\hline 529.07 & 174 & 7 & 0.0210 & 301.40 & 9.04 & 20.96 & 0.0246 & 0.0745 & 0.8458 \\
\hline 531.86 & 175 & 7 & 0.0208 & 305.08 & 9.15 & 20.85 & 0.0254 & 0.0719 & 0.8423 \\
\hline 534.68 & 176 & 7 & 0.0207 & 308.79 & 9.26 & 20.74 & 0.0261 & 0.0693 & 0.8388 \\
\hline 537.51 & 177 & 7 & 0.0206 & 312.52 & 9.38 & 20.62 & 0.0269 & 0.0668 & 0.8353 \\
\hline 540.36 & 178 & 7 & 0.0205 & 316.26 & 9.49 & 20.51 & 0.0277 & 0.0643 & 0.8318 \\
\hline 543.22 & 179 & 7 & 0.0204 & 320.03 & 9.60 & 20.40 & 0.0285 & 0.0620 & 0.8282 \\
\hline 546.10 & 180 & 7 & 0.0203 & 323.82 & 9.71 & 20.29 & 0.0293 & 0.0597 & 0.8246 \\
\hline 549.00 & 181 & 7 & 0.0202 & 327.63 & 9.83 & 20.17 & 0.0302 & 0.0575 & 0.8210 \\
\hline 551.91 & 182 & 7 & 0.0201 & 331.46 & 9.94 & 20.06 & 0.0310 & 0.0554 & 0.8173 \\
\hline 554.83 & 183 & 7 & 0.0199 & 335.31 & 10.06 & 19.94 & 0.0319 & 0.0534 & 0.8136 \\
\hline 557.77 & 184 & 7 & 0.0198 & 339.18 & 10.18 & 19.82 & 0.0328 & 0.0514 & 0.8098 \\
\hline 504.40 & 184 & 8 & 0.0219 & 268.95 & 8.07 & 21.93 & 0.0124 & 0.0971 & 0.8959 \\
\hline 560.73 & 185 & 7 & 0.0197 & 343.06 & 10.29 & 19.71 & 0.0337 & 0.0495 & 0.8061 \\
\hline 506.68 & 185 & 8 & 0.0218 & 271.95 & 8.16 & 21.84 & 0.0128 & 0.0939 & 0.8933 \\
\hline 563.69 & 186 & 7 & 0.0196 & 346.96 & 10.41 & 19.59 & 0.0346 & 0.0476 & 0.8023 \\
\hline 508.98 & 186 & 8 & 0.0218 & 274.97 & 8.25 & 21.75 & 0.0132 & 0.0908 & 0.8907 \\
\hline 566.67 & 187 & 7 & 0.0195 & 350.88 & 10.53 & 19.47 & 0.0356 & 0.0458 & 0.7984 \\
\hline
\end{tabular}


Table 1: Continued.

\begin{tabular}{|c|c|c|c|c|c|c|c|c|c|}
\hline $\mathrm{TC}$ & $n$ & $c$ & AOQ & ATI & $D_{d}$ & $D_{n}$ & $1-P_{a}(\mathrm{AQL})$ & $P_{a}(\mathrm{LTPD})$ & $P_{a}(p)$ \\
\hline 511.30 & 187 & 8 & 0.0217 & 278.02 & 8.34 & 21.66 & 0.0136 & 0.0877 & 0.8880 \\
\hline 569.66 & 188 & 7 & 0.0194 & 354.82 & 10.64 & 19.36 & 0.0365 & 0.0441 & 0.7946 \\
\hline 513.63 & 188 & 8 & 0.0216 & 281.09 & 8.43 & 21.57 & 0.0141 & 0.0848 & 0.8854 \\
\hline 572.66 & 189 & 7 & 0.0192 & 358.77 & 10.76 & 19.24 & 0.0375 & 0.0424 & 0.7907 \\
\hline 515.98 & 189 & 8 & 0.0215 & 284.19 & 8.53 & 21.47 & 0.0145 & 0.0819 & 0.8826 \\
\hline 575.68 & 190 & 7 & 0.0191 & 362.74 & 10.88 & 19.12 & 0.0385 & 0.0408 & 0.7867 \\
\hline 518.35 & 190 & 8 & 0.0214 & 287.30 & 8.62 & 21.38 & 0.0150 & 0.0792 & 0.8799 \\
\hline 578.71 & 191 & 7 & 0.0190 & 366.72 & 11.00 & 19.00 & 0.0395 & 0.0392 & 0.7828 \\
\hline 520.74 & 191 & 8 & 0.0213 & 290.44 & 8.71 & 21.29 & 0.0154 & 0.0765 & 0.8771 \\
\hline 581.74 & 192 & 7 & 0.0189 & 370.71 & 11.12 & 18.88 & 0.0405 & 0.0377 & 0.7788 \\
\hline 523.14 & 192 & 8 & 0.0212 & 293.61 & 8.81 & 21.19 & 0.0159 & 0.0739 & 0.8742 \\
\hline 584.79 & 193 & 7 & 0.0188 & 374.72 & 11.24 & 18.76 & 0.0415 & 0.0363 & 0.7748 \\
\hline 525.56 & 193 & 8 & 0.0211 & 296.79 & 8.90 & 21.10 & 0.0164 & 0.0714 & 0.8714 \\
\hline 587.85 & 194 & 7 & 0.0186 & 378.74 & 11.36 & 18.64 & 0.0426 & 0.0349 & 0.7708 \\
\hline 528.00 & 194 & 8 & 0.0210 & 300.00 & 9.00 & 21.00 & 0.0169 & 0.0689 & 0.8685 \\
\hline 590.91 & 195 & 7 & 0.0185 & 382.78 & 11.48 & 18.52 & 0.0437 & 0.0335 & 0.7667 \\
\hline 530.45 & 195 & 8 & 0.0209 & 303.23 & 9.10 & 20.90 & 0.0174 & 0.0665 & 0.8656 \\
\hline 593.99 & 196 & 7 & 0.0184 & 386.83 & 11.60 & 18.40 & 0.0448 & 0.0322 & 0.7627 \\
\hline 532.92 & 196 & 8 & 0.0208 & 306.47 & 9.19 & 20.81 & 0.0180 & 0.0642 & 0.8626 \\
\hline 597.07 & 197 & 7 & 0.0183 & 390.88 & 11.73 & 18.27 & 0.0459 & 0.0309 & 0.7586 \\
\hline 535.41 & 197 & 8 & 0.0207 & 309.74 & 9.29 & 20.71 & 0.0185 & 0.0620 & 0.8596 \\
\hline 600.16 & 198 & 7 & 0.0182 & 394.95 & 11.85 & 18.15 & 0.0470 & 0.0297 & 0.7544 \\
\hline 537.91 & 198 & 8 & 0.0206 & 313.04 & 9.39 & 20.61 & 0.0190 & 0.0598 & 0.8566 \\
\hline 603.26 & 199 & 7 & 0.0180 & 399.03 & 11.97 & 18.03 & 0.0482 & 0.0285 & 0.7503 \\
\hline 540.42 & 199 & 8 & 0.0205 & 316.35 & 9.49 & 20.51 & 0.0196 & 0.0577 & 0.8535 \\
\hline 606.37 & 200 & 7 & 0.0179 & 403.12 & 12.09 & 17.91 & 0.0493 & 0.0274 & 0.7461 \\
\hline 542.95 & 200 & 8 & 0.0204 & 319.68 & 9.59 & 20.41 & 0.0202 & 0.0556 & 0.8504 \\
\hline 545.50 & 201 & 8 & 0.0203 & 323.03 & 9.69 & 20.31 & 0.0208 & 0.0537 & 0.8473 \\
\hline 503.07 & 201 & 9 & 0.0220 & 267.19 & 8.02 & 21.98 & 0.0077 & 0.0978 & 0.9172 \\
\hline 548.06 & 202 & 8 & 0.0202 & 326.40 & 9.79 & 20.21 & 0.0214 & 0.0518 & 0.8441 \\
\hline 505.03 & 202 & 9 & 0.0219 & 269.78 & 8.09 & 21.91 & 0.0080 & 0.0947 & 0.9151 \\
\hline 550.64 & 203 & 8 & 0.0201 & 329.79 & 9.89 & 20.11 & 0.0220 & 0.0499 & 0.8409 \\
\hline 507.02 & 203 & 9 & 0.0218 & 272.39 & 8.17 & 21.83 & 0.0083 & 0.0917 & 0.9129 \\
\hline 553.23 & 204 & 8 & 0.0200 & 333.19 & 10.00 & 20.00 & 0.0226 & 0.0481 & 0.8377 \\
\hline 509.02 & 204 & 9 & 0.0217 & 275.03 & 8.25 & 21.75 & 0.0085 & 0.0888 & 0.9108 \\
\hline 555.83 & 205 & 8 & 0.0199 & 336.62 & 10.10 & 19.90 & 0.0232 & 0.0464 & 0.8344 \\
\hline 511.04 & 205 & 9 & 0.0217 & 277.68 & 8.33 & 21.67 & 0.0088 & 0.0859 & 0.9086 \\
\hline
\end{tabular}

a defective item which is released to the market. For an incoming raw material, this will be the attendant cost when a defective item is released for production use.

The economic sampling plan can be found through the following mathematical model:

$$
\begin{array}{ll}
\text { Minimize } & \mathrm{TC}=C_{i} \cdot \mathrm{ATI}+C_{f} \cdot D_{d}+C_{o} \cdot D_{n} \\
\text { Subject to } & 1-p_{a}(\mathrm{AQL}) \leq \alpha \\
& p_{a}(\mathrm{LTPD}) \leq \beta
\end{array}
$$


Table 2: Optimal single sampling plan as a function of the product quality $p$ (other input parameters are given as the base set).

\begin{tabular}{lcccccccccc}
\hline$p$ & TC & $n$ & $c$ & AOQ & ATI & $D_{d}$ & $D_{n}$ & $1-P_{a}(\mathrm{AQL})$ & $P_{a}(\mathrm{LTPD})$ & $P_{a}(p)$ \\
\hline 0.01 & 222.25 & 131 & 5 & 0.0087 & 132.88 & 1.33 & 8.67 & 0.0487 & 0.0974 & 0.9978 \\
0.02 & 345.61 & 131 & 5 & 0.0165 & 173.34 & 3.47 & 16.53 & 0.0487 & 0.0974 & 0.9513 \\
0.03 & 503.07 & 201 & 9 & 0.0220 & 267.19 & 8.02 & 21.98 & 0.0077 & 0.0978 & 0.9172 \\
0.04 & 676.49 & 268 & 13 & 0.0237 & 406.60 & 16.26 & 23.74 & 0.0012 & 0.0995 & 0.8106 \\
0.05 & 862.78 & 334 & 17 & 0.0198 & 604.63 & 30.23 & 19.77 & 0.0002 & 0.0997 & 0.5937 \\
0.06 & 1020.20 & 301 & 15 & 0.0115 & 808.08 & 48.48 & 11.52 & 0.0004 & 0.1000 & 0.2746 \\
0.07 & 1102.75 & 131 & 5 & 0.0059 & 915.35 & 64.07 & 5.93 & 0.0487 & 0.0974 & 0.0974 \\
0.08 & 1146.08 & 131 & 5 & 0.0031 & 961.33 & 76.91 & 3.09 & 0.0487 & 0.0974 & 0.0445 \\
0.09 & 1175.40 & 131 & 5 & 0.0015 & 983.57 & 88.52 & 1.48 & 0.0487 & 0.0974 & 0.0189 \\
0.10 & 1198.69 & 131 & 5 & 0.0007 & 993.44 & 99.34 & 0.66 & 0.0487 & 0.0974 & 0.0075 \\
0.11 & 1219.70 & 131 & 5 & 0.0003 & 997.52 & 109.73 & 0.27 & 0.0487 & 0.0974 & 0.0029 \\
0.12 & 1239.96 & 131 & 5 & 0.0001 & 999.11 & 119.89 & 0.11 & 0.0487 & 0.0974 & 0.0010 \\
0.13 & 1260.00 & 228 & 8 & 0.0000 & 1000.00 & 130.00 & 0.00 & 0.0414 & 0.0191 & 0.0000 \\
0.14 & 1280.00 & 218 & 8 & 0.0000 & 1000.00 & 140.00 & 0.00 & 0.0326 & 0.0284 & 0.0000 \\
0.15 & 1300.00 & 198 & 7 & 0.0000 & 1000.00 & 150.00 & 0.00 & 0.0470 & 0.0297 & 0.0000 \\
0.16 & 1320.00 & 183 & 7 & 0.0000 & 1000.00 & 160.00 & 0.00 & 0.0319 & 0.0534 & 0.0000 \\
0.17 & 1340.00 & 193 & 7 & 0.0000 & 1000.00 & 170.00 & 0.00 & 0.0415 & 0.0363 & 0.0000 \\
0.18 & 1360.00 & 187 & 7 & 0.0000 & 1000.00 & 180.00 & 0.00 & 0.0356 & 0.0458 & 0.0000 \\
0.19 & 1380.00 & 152 & 6 & 0.0000 & 1000.00 & 190.00 & 0.00 & 0.0340 & 0.0867 & 0.0000 \\
0.20 & 1400.00 & 131 & 5 & 0.0000 & 1000.00 & 200.00 & 0.00 & 0.0487 & 0.0974 & 0.0000 \\
\hline
\end{tabular}

Note that for the cases of the export of the seedlings of Phalaenopsis from Taiwan, if the defective products are scrapped at the seaport, the internal failure cost would be the lost profit (i.e., revenue-production cost-transportation cost (from Taiwan to the USA)). If the defective products are returned to Taiwan, the internal failure cost would be the lost profit plus the transportation cost (from the USA to Taiwan) subtract the salvage value when the defective products arrive in Taiwan. If the defective products are sold at a reduced price, the internal failure cost would be calculated as follows: revenue (the original selling price) - the production cost - transportation cost (from Taiwan to The Netherlands) — the reduced selling price.

Note that if the cost of an outgoing defective item $C_{o}$ is relatively high in comparison to the inspection cost per item $C_{i}$ and the internal failure cost per item $C_{f}$, then the optimal sampling plan is to have a $100 \%$ inspection of the entire lot. If $C_{o}$ is high, then in order to minimize the total cost TC, the defective items not detected $D_{n}$ should be as small as possible. Since $D_{n}=p_{a}(p)(N-n) p$, if the sample size $n$ equals the lot size $N(100 \%$ inspection), then $D_{n}=0$. On the contrary, if the inspection cost per item $C_{i}$ is relatively high in comparison to the internal failure cost per item $C_{f}$ and the cost of an outgoing defective item $C_{o}$, then the optimal sampling plan is to have zero inspection without take into consideration the producer's and the consumer's risk requirements. However, with zero inspection, the consumer's risk would be high and may not be acceptable to the consumer. 
Table 3: Optimal single sampling plan as a function of the inspection $\operatorname{cost} C_{i}$ (other input parameters are given as the base set).

\begin{tabular}{ccccccccccc}
\hline$C_{i}$ & TC & $n$ & $c$ & AOQ & ATI & $D_{d}$ & $D_{n}$ & $1-P_{a}(\mathrm{AQL})$ & $P_{a}(\mathrm{LTPD})$ & $P_{a}(p)$ \\
\hline 0.1 & 160.00 & 1000 & 28 & 0.0000 & 1000.00 & 30.00 & 0.00 & 0.0329 & 0.0000 & 0.4009 \\
0.2 & 260.00 & 1000 & 28 & 0.0000 & 1000.00 & 30.00 & 0.00 & 0.0329 & 0.0000 & 0.4009 \\
0.3 & 316.03 & 201 & 9 & 0.0220 & 267.19 & 8.02 & 21.98 & 0.0077 & 0.0978 & 0.9172 \\
0.4 & 342.75 & 201 & 9 & 0.0220 & 267.19 & 8.02 & 21.98 & 0.0077 & 0.0978 & 0.9172 \\
0.5 & 369.47 & 201 & 9 & 0.0220 & 267.19 & 8.02 & 21.98 & 0.0077 & 0.0978 & 0.9172 \\
1.0 & 503.07 & 201 & 9 & 0.0220 & 267.19 & 8.02 & 21.98 & 0.0077 & 0.0978 & 0.9172 \\
1.5 & 636.66 & 201 & 9 & 0.0220 & 267.19 & 8.02 & 21.98 & 0.0077 & 0.0978 & 0.9172 \\
2.0 & 770.26 & 201 & 9 & 0.0220 & 267.19 & 8.02 & 21.98 & 0.0077 & 0.0978 & 0.9172 \\
2.5 & 903.85 & 201 & 9 & 0.0220 & 267.19 & 8.02 & 21.98 & 0.0077 & 0.0978 & 0.9172 \\
3.0 & 1037.45 & 201 & 9 & 0.0220 & 267.19 & 8.02 & 21.98 & 0.0077 & 0.0978 & 0.9172 \\
3.5 & 1171.05 & 201 & 9 & 0.0220 & 267.19 & 8.02 & 21.98 & 0.0077 & 0.0978 & 0.9172 \\
4.0 & 1304.64 & 201 & 9 & 0.0220 & 267.19 & 8.02 & 21.98 & 0.0077 & 0.0978 & 0.9172 \\
4.5 & 1438.24 & 201 & 9 & 0.0220 & 267.19 & 8.02 & 21.98 & 0.0077 & 0.0978 & 0.9172 \\
5.0 & 1571.84 & 201 & 9 & 0.0220 & 267.19 & 8.02 & 21.98 & 0.0077 & 0.0978 & 0.9172 \\
5.5 & 1705.43 & 201 & 9 & 0.0220 & 267.19 & 8.02 & 21.98 & 0.0077 & 0.0978 & 0.9172 \\
6.0 & 1839.03 & 201 & 9 & 0.0220 & 267.19 & 8.02 & 21.98 & 0.0077 & 0.0978 & 0.9172 \\
6.5 & 1972.62 & 201 & 9 & 0.0220 & 267.19 & 8.02 & 21.98 & 0.0077 & 0.0978 & 0.9172 \\
7.0 & 2106.22 & 201 & 9 & 0.0220 & 267.19 & 8.02 & 21.98 & 0.0077 & 0.0978 & 0.9172 \\
7.5 & 2239.82 & 201 & 9 & 0.0220 & 267.19 & 8.02 & 21.98 & 0.0077 & 0.0978 & 0.9172 \\
8.0 & 2373.41 & 201 & 9 & 0.0220 & 267.19 & 8.02 & 21.98 & 0.0077 & 0.0978 & 0.9172 \\
8.5 & 2507.01 & 201 & 9 & 0.0220 & 267.19 & 8.02 & 21.98 & 0.0077 & 0.0978 & 0.9172 \\
9.0 & 2640.60 & 201 & 9 & 0.0220 & 267.19 & 8.02 & 21.98 & 0.0077 & 0.0978 & 0.9172 \\
9.5 & 2774.20 & 201 & 9 & 0.0220 & 267.19 & 8.02 & 21.98 & 0.0077 & 0.0978 & 0.9172 \\
10.0 & 2907.80 & 201 & 9 & 0.0220 & 267.19 & 8.02 & 21.98 & 0.0077 & 0.0978 & 0.9172 \\
\hline & & & & & & & & &
\end{tabular}

\section{Numerical Examples and Discussion}

For the purpose of our illustration, we consider the following set of input parameters: $N=1,000, \mathrm{AQL}=0.02, \mathrm{LTPD}=0.07, \alpha=0.05, \beta=0.10, p=0.03, C_{i}=1.0, C_{f}=$ 2.0, and $C_{o}=10$. We use MATLAB computer software to obtain all the single sampling plans with sample size $n$ less than or equal to 1000 that satisfy both the producer's and consumer's quality and risk requirements. To indicate the performance measurements, Table 1 lists all the single sampling plans for $n$ up to 205. From Table 1, one can see that both the producer's risk $\left(1-p_{a}(\mathrm{AQL})\right)$ and average total inspection (ATI) increase, and the consumer's risk $p_{a}(\mathrm{LTPD})$ decreases as $n$ increases and $c$ remains unchanged; on the contrary, both the producer's risk and average total inspection decrease, and the consumer's risk increases as $c$ increases and $n$ remains unchanged. Based on the previous input parameters, the optimal sampling plan is $n=201$ and $c=9$ with the total cost TC $=503.07$. Note that without constraints (2.6) and (2.7), the optimal decision for the producer is to have zero inspection $(n=0)$ with the total cost TC $=300$, the producer's risk $\alpha=0$, and the consumer's risk $\beta=1$, which obviously is not acceptable to the consumer. This example indicates that without integrating the producer's and the consumer's risk requirements into the economic design of the acceptance sampling plans, the plan obtained by the model, although minimizing the producer's and the consumer's total quality cost, may not be acceptable to the consumer. 
Advances in Decision Sciences

Table 4

(a) Optimal single sampling plan as a function of the internal failure $\operatorname{cost} C_{f}$ (other input parameters are given as the base set)

\begin{tabular}{ccccccccccc}
\hline$C_{f}$ & TC & $n$ & $c$ & AOQ & ATI & $D_{d}$ & $D_{n}$ & $1-P_{a}(\mathrm{AQL})$ & $P_{a}(\mathrm{LTPD})$ & $P_{a}(p)$ \\
\hline 0.0 & 487.03 & 201 & 9 & 0.0220 & 267.19 & 8.02 & 21.98 & 0.0077 & 0.0978 & 0.9172 \\
0.5 & 491.04 & 201 & 9 & 0.0220 & 267.19 & 8.02 & 21.98 & 0.0077 & 0.0978 & 0.9172 \\
1.0 & 495.05 & 201 & 9 & 0.0220 & 267.19 & 8.02 & 21.98 & 0.0077 & 0.0978 & 0.9172 \\
1.5 & 499.06 & 201 & 9 & 0.0220 & 267.19 & 8.02 & 21.98 & 0.0077 & 0.0978 & 0.9172 \\
2.0 & 503.07 & 201 & 9 & 0.0220 & 267.19 & 8.02 & 21.98 & 0.0077 & 0.0978 & 0.9172 \\
2.5 & 507.07 & 201 & 9 & 0.0220 & 267.19 & 8.02 & 21.98 & 0.0077 & 0.0978 & 0.9172 \\
3.0 & 511.08 & 201 & 9 & 0.0220 & 267.19 & 8.02 & 21.98 & 0.0077 & 0.0978 & 0.9172 \\
3.5 & 515.09 & 201 & 9 & 0.0220 & 267.19 & 8.02 & 21.98 & 0.0077 & 0.0978 & 0.9172 \\
4.0 & 519.10 & 201 & 9 & 0.0220 & 267.19 & 8.02 & 21.98 & 0.0077 & 0.0978 & 0.9172 \\
4.5 & 523.11 & 201 & 9 & 0.0220 & 267.19 & 8.02 & 21.98 & 0.0077 & 0.0978 & 0.9172 \\
5.0 & 527.11 & 201 & 9 & 0.0220 & 267.19 & 8.02 & 21.98 & 0.0077 & 0.0978 & 0.9172 \\
5.5 & 531.12 & 201 & 9 & 0.0220 & 267.19 & 8.02 & 21.98 & 0.0077 & 0.0978 & 0.9172 \\
6.0 & 535.13 & 201 & 9 & 0.0220 & 267.19 & 8.02 & 21.98 & 0.0077 & 0.0978 & 0.9172 \\
6.5 & 539.14 & 201 & 9 & 0.0220 & 267.19 & 8.02 & 21.98 & 0.0077 & 0.0978 & 0.9172 \\
7.0 & 543.14 & 201 & 9 & 0.0220 & 267.19 & 8.02 & 21.98 & 0.0077 & 0.0978 & 0.9172 \\
7.5 & 547.15 & 201 & 9 & 0.0220 & 267.19 & 8.02 & 21.98 & 0.0077 & 0.0978 & 0.9172 \\
8.0 & 551.16 & 201 & 9 & 0.0220 & 267.19 & 8.02 & 21.98 & 0.0077 & 0.0978 & 0.9172 \\
8.5 & 555.17 & 201 & 9 & 0.0220 & 267.19 & 8.02 & 21.98 & 0.0077 & 0.0978 & 0.9172 \\
9.0 & 559.18 & 201 & 9 & 0.0220 & 267.19 & 8.02 & 21.98 & 0.0077 & 0.0978 & 0.9172 \\
9.5 & 563.18 & 201 & 9 & 0.0220 & 267.19 & 8.02 & 21.98 & 0.0077 & 0.0978 & 0.9172 \\
10.0 & 567.19 & 201 & 9 & 0.0220 & 267.19 & 8.02 & 21.98 & 0.0077 & 0.0978 & 0.9172 \\
\hline
\end{tabular}

(b) Optimal single sampling plan as a function of the internal failure cost $C_{f}$ (with $C_{i}=0.2$ and other input parameters are given as the base set).

\begin{tabular}{ccccccccccc}
\hline$C_{f}$ & TC & $n$ & $c$ & AOQ & ATI & $D_{d}$ & $D_{n}$ & $1-P_{a}(\mathrm{AQL})$ & $P_{a}(\mathrm{LTPD})$ & $P_{a}(p)$ \\
\hline 0.0 & 200.00 & 1000 & 28 & 0.0000 & 1000.00 & 30.00 & 0.00 & 0.0329 & 0.0000 & 0.4009 \\
0.5 & 215.00 & 1000 & 28 & 0.0000 & 1000.00 & 30.00 & 0.00 & 0.0329 & 0.0000 & 0.4009 \\
1.0 & 230.00 & 1000 & 28 & 0.0000 & 1000.00 & 30.00 & 0.00 & 0.0329 & 0.0000 & 0.4009 \\
1.5 & 245.00 & 1000 & 28 & 0.0000 & 1000.00 & 30.00 & 0.00 & 0.0329 & 0.0000 & 0.4009 \\
2.0 & 260.00 & 1000 & 28 & 0.0000 & 1000.00 & 30.00 & 0.00 & 0.0329 & 0.0000 & 0.4009 \\
2.5 & 275.00 & 1000 & 28 & 0.0000 & 1000.00 & 30.00 & 0.00 & 0.0329 & 0.0000 & 0.4009 \\
3.0 & 290.00 & 1000 & 28 & 0.0000 & 1000.00 & 30.00 & 0.00 & 0.0329 & 0.0000 & 0.4009 \\
3.5 & 301.34 & 201 & 9 & 0.0220 & 267.19 & 8.02 & 21.98 & 0.0077 & 0.0978 & 0.9172 \\
4.0 & 305.34 & 201 & 9 & 0.0220 & 267.19 & 8.02 & 21.98 & 0.0077 & 0.0978 & 0.9172 \\
4.5 & 309.35 & 201 & 9 & 0.0220 & 267.19 & 8.02 & 21.98 & 0.0077 & 0.0978 & 0.9172 \\
5.0 & 313.36 & 201 & 9 & 0.0220 & 267.19 & 8.02 & 21.98 & 0.0077 & 0.0978 & 0.9172 \\
5.5 & 317.37 & 201 & 9 & 0.0220 & 267.19 & 8.02 & 21.98 & 0.0077 & 0.0978 & 0.9172 \\
6.0 & 321.38 & 201 & 9 & 0.0220 & 267.19 & 8.02 & 21.98 & 0.0077 & 0.0978 & 0.9172 \\
6.5 & 325.38 & 201 & 9 & 0.0220 & 267.19 & 8.02 & 21.98 & 0.0077 & 0.0978 & 0.9172 \\
7.0 & 329.39 & 201 & 9 & 0.0220 & 267.19 & 8.02 & 21.98 & 0.0077 & 0.0978 & 0.9172 \\
7.5 & 333.40 & 201 & 9 & 0.0220 & 267.19 & 8.02 & 21.98 & 0.0077 & 0.0978 & 0.9172 \\
8.0 & 337.41 & 201 & 9 & 0.0220 & 267.19 & 8.02 & 21.98 & 0.0077 & 0.0978 & 0.9172 \\
8.5 & 341.41 & 201 & 9 & 0.0220 & 267.19 & 8.02 & 21.98 & 0.0077 & 0.0978 & 0.9172 \\
9.0 & 345.42 & 201 & 9 & 0.0220 & 267.19 & 8.02 & 21.98 & 0.0077 & 0.0978 & 0.9172 \\
9.5 & 349.43 & 201 & 9 & 0.0220 & 267.19 & 8.02 & 21.98 & 0.0077 & 0.0978 & 0.9172 \\
10.0 & 353.44 & 201 & 9 & 0.0220 & 267.19 & 8.02 & 21.98 & 0.0077 & 0.0978 & 0.9172 \\
\hline & & & & & & & & &
\end{tabular}


Table 5: Optimal single sampling plan as a function of the post sale failure $\operatorname{cost} C_{o}$ (other input parameters are given as the base set).

\begin{tabular}{lcccccccccc}
\hline$C_{o}$ & TC & $n$ & $c$ & AOQ & ATI & $D_{d}$ & $D_{n}$ & $1-P_{a}(\mathrm{AQL})$ & $P_{a}(\mathrm{LTPD})$ & $P_{a}(p)$ \\
\hline 5 & 393.14 & 201 & 9 & 0.0220 & 267.19 & 8.02 & 21.98 & 0.0077 & 0.0978 & 0.9172 \\
10 & 503.07 & 201 & 9 & 0.0220 & 267.19 & 8.02 & 21.98 & 0.0077 & 0.0978 & 0.9172 \\
15 & 612.99 & 201 & 9 & 0.0220 & 267.19 & 8.02 & 21.98 & 0.0077 & 0.0978 & 0.9172 \\
20 & 722.91 & 201 & 9 & 0.0220 & 267.19 & 8.02 & 21.98 & 0.0077 & 0.0978 & 0.9172 \\
25 & 832.83 & 201 & 9 & 0.0220 & 267.19 & 8.02 & 21.98 & 0.0077 & 0.0978 & 0.9172 \\
30 & 942.75 & 201 & 9 & 0.0220 & 267.19 & 8.02 & 21.98 & 0.0077 & 0.0978 & 0.9172 \\
35 & 1052.67 & 201 & 9 & 0.0220 & 267.19 & 8.02 & 21.98 & 0.0077 & 0.0978 & 0.9172 \\
40 & 1060.00 & 1000 & 28 & 0.0000 & 1000.00 & 30.00 & 0.00 & 0.0329 & 0.0000 & 0.4009 \\
45 & 1060.00 & 1000 & 28 & 0.0000 & 1000.00 & 30.00 & 0.00 & 0.0329 & 0.0000 & 0.4009 \\
50 & 1060.00 & 1000 & 28 & 0.0000 & 1000.00 & 30.00 & 0.00 & 0.0329 & 0.0000 & 0.4009 \\
55 & 1060.00 & 1000 & 28 & 0.0000 & 1000.00 & 30.00 & 0.00 & 0.0329 & 0.0000 & 0.4009 \\
60 & 1060.00 & 1000 & 28 & 0.0000 & 1000.00 & 30.00 & 0.00 & 0.0329 & 0.0000 & 0.4009 \\
65 & 1060.00 & 1000 & 28 & 0.0000 & 1000.00 & 30.00 & 0.00 & 0.0329 & 0.0000 & 0.4009 \\
70 & 1060.00 & 1000 & 28 & 0.0000 & 1000.00 & 30.00 & 0.00 & 0.0329 & 0.0000 & 0.4009 \\
75 & 1060.00 & 1000 & 28 & 0.0000 & 1000.00 & 30.00 & 0.00 & 0.0329 & 0.0000 & 0.4009 \\
80 & 1060.00 & 1000 & 28 & 0.0000 & 1000.00 & 30.00 & 0.00 & 0.0329 & 0.0000 & 0.4009 \\
85 & 1060.00 & 1000 & 28 & 0.0000 & 1000.00 & 30.00 & 0.00 & 0.0329 & 0.0000 & 0.4009 \\
90 & 1060.00 & 1000 & 28 & 0.0000 & 1000.00 & 30.00 & 0.00 & 0.0329 & 0.0000 & 0.4009 \\
95 & 1060.00 & 1000 & 28 & 0.0000 & 1000.00 & 30.00 & 0.00 & 0.0329 & 0.0000 & 0.4009 \\
100 & 1060.00 & 1000 & 28 & 0.0000 & 1000.00 & 30.00 & 0.00 & 0.0329 & 0.0000 & 0.4009 \\
\hline
\end{tabular}

Figure 2 shows the total cost with different sampling plans that satisfy both the producer's and consumer's quality and risk requirements (i.e., AQL $=0.02$, LTPD $=$ $0.07, \alpha=0.05, \beta=0.10$ ). For a given $c$ value, as $n$ increases, the total cost increases. However, when $n$ increases or decreases to a certain value, the sampling plan becomes infeasible (i.e., the consumer's or the producer's risk becomes too big).

Table 2 shows the sensitivity analyses of the optimal single sampling with different levels of $p$. As $p$ increases, the optimal sample size first increases and then decreases. For $p \geq 0.13$, the optimal sampling plan will have a near zero probability of accepting the lot, resulting in a $100 \%$ inspection of the entire lot. As a result, all the defective products will be detected and replaced (ATI $=1000$ and $A O Q=0)$.

Table 3 shows the sensitivity analysis of the inspection cost $C_{i}$. If $C_{i} \leq 0.2$, the inspection cost is relatively low compared to the failure costs $\left(C_{f}\right.$ and $\left.C_{o}\right)$. Therefore, the optimal sampling plan is to have a $100 \%$ inspection of the entire lot. For $0.3 \leq C_{i} \leq 10$, the optimal sampling plans remain at $n=201$ and $c=9$.

As shown in Table 4(a), one can see that the internal failure cost $C_{f}$ is relatively insensitive to the optimal sampling plan. However, when the inspection $\operatorname{cost} C_{i}$ is small, for example, $C_{i}=0.2$ (see Table $4(\mathrm{~b})$ ), the internal failure $\operatorname{cost} C_{f}$ has an effect on the optimal sampling plan.

Table 5 shows the sensitivity analysis of the postsale failure cost $C_{o}$. For $C_{o} \leq 35$, the optimal sampling plans remain to be $n=201$ and $c=9$. However, when $C_{o} \geq 40$, the optimal sampling plan changes to have a $100 \%$ inspection of the entire lot. 


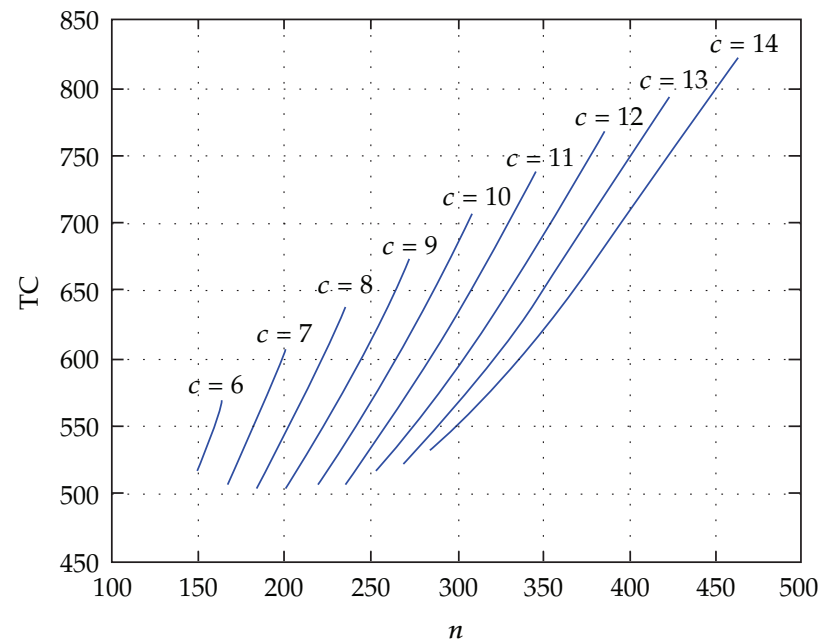

Figure 2: Total cost (TC) versus sample size $(n)$ at different $c$ when $C_{i}=1, C_{f}=2$, and $C_{o}=10$.

\section{Conclusions}

There are many ways to determine an acceptance sampling plan. However, all of them are either settled on a noneconomic basis or did not take into consideration the producer's and consumer's quality and risk requirements. In this paper, we developed a mathematical model for a two-stage supply chain that can help the producer and the consumer to find a single sampling plan that minimizes the producer's and the consumer's total quality cost (inspection and failure costs) and satisfies both the producer's and consumer's quality and risk requirements. From the numerical analyses, we see that the optimal sampling plan is very sensitive to the producer's product quality. The product inspection, internal failure, and postsale failure costs also have an effect on the choice of the economic sampling plan. The results presented in this paper can be further extended to develop models for double or multiple sampling plans. The mathematical model and computer program for determining an optimal double or multiple sampling plans are more complicated. The research work is now being undertaken.

\section{References}

[1] O. Kabak and F. Ülengin, "Possibilistic linear-programming approach for supply chain networking decisions," European Journal of Operational Research, vol. 209, no. 3, pp. 253-264, 2011.

[2] M. Cao and Q. Zhang, "Supply chain collaborative advantage: a firm's perspective," International Journal of Production Economics, vol. 128, no. 1, pp. 358-367, 2010.

[3] S. T. Foster Jr., "Towards an understanding of supply chain quality management," Journal of Operations Management, vol. 26, no. 4, pp. 461-467, 2008.

[4] ANSI/ASQ Z1.4 and Z1.9-2008, Sampling Procedures and Package, American National Standard Institute, 2008.

[5] Military Standard (MIL-STD-105E), Sampling Procedures and Tables for Inspection by Attributes, U.S. Department of Defense, Washington, DC, USA, 1989.

[6] H. F. Dodge and H. G. Romig, Sampling Inspection Tables, John Wiley \& Sons, New York, NY, USA, 2nd edition, 1998

[7] Sampling Plan Analyzer 2.0, Taylor Enterprises, Libertyville, Ill, USA, 2010.

[8] Acceptance Sampling for Attributesm TP105, H \& H Servicco, North St. Paul, Minn, USA, 2010. 
[9] International Organization for Standardization. Sampling Procedures and Tables for Inspection by Attributes, ISO 2859, Geneva, Switzerland, 1974.

[10] Y. Nikolaidis and G. Nenes, "Economic evaluation of ISO 2859 acceptance sampling plans used with rectifying inspection of rejected lots," Quality Engineering, vol. 21, no. 1, pp. 10-23, 2009.

[11] G. B. Wetherill and W. K. Chiu, "A review of acceptance sampling schemes with emphasis on the economic aspect," International Statistical Review, vol. 43, no. 2, pp. 191-210, 1975.

[12] G. Tagaras, "Economic acceptance sampling plans by variables with quadratic quality cost," IIE Transactions, vol. 26, no. 6, pp. 29-36, 1994.

[13] W. G. Ferrell Jr. and A. Chhoker, "Design of economically optimal acceptance sampling plans with inspection error," Computers and Operations Research, vol. 29, no. 10, pp. 1283-1300, 2002.

[14] C. González and G. Palomo, "Bayesian acceptance sampling plans following economic criteria: an application to paper pulp manufacturing," Journal of Applied Statistics, vol. 30, no. 3, pp. 319-333, 2003.

[15] D. C. Montgomery, Introduction to Statistical Quality Control, John Wiley \& Sons, New York, NY, USA, 4th edition, 2000.

[16] L. F. Hsu and C. S. Tapiero, "An economic model for determining the optimal quality and process control policy in a queue-like production system," International Journal of Production Research, vol. 28, no. 8, pp. 1447-1457, 1990. 


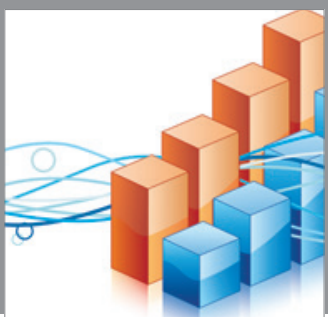

Advances in

Operations Research

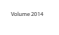

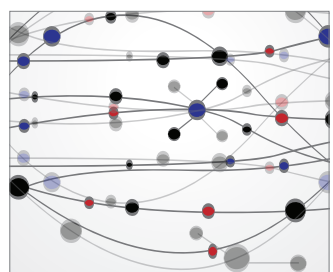

\section{The Scientific} World Journal
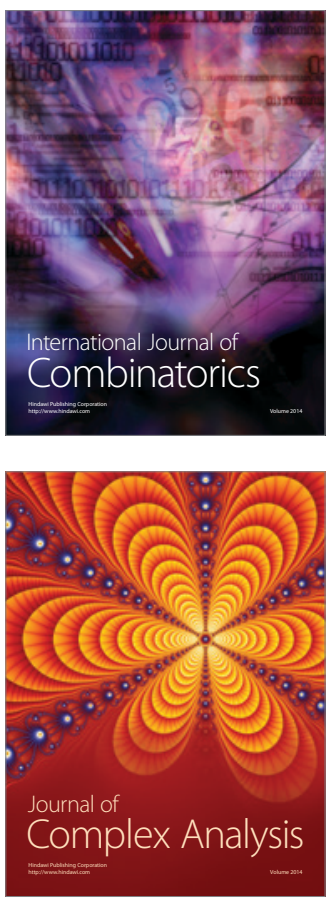

International Journal of

Mathematics and

Mathematical

Sciences
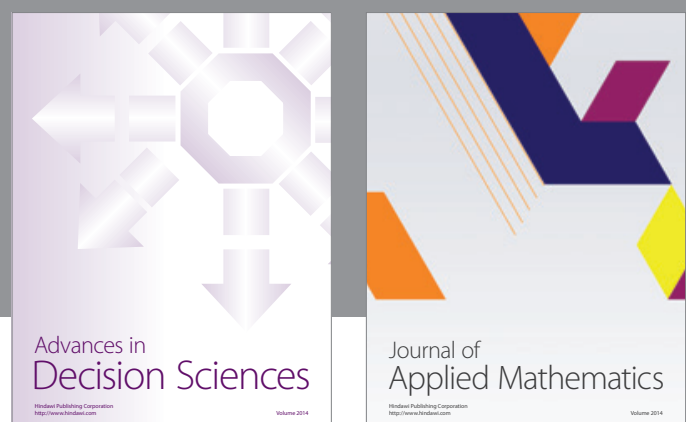

Journal of

Applied Mathematics
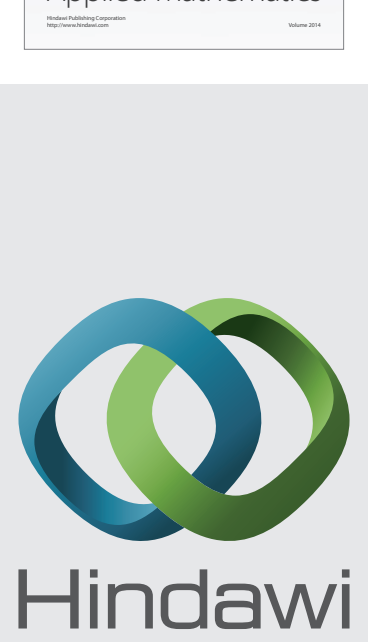

Submit your manuscripts at http://www.hindawi.com
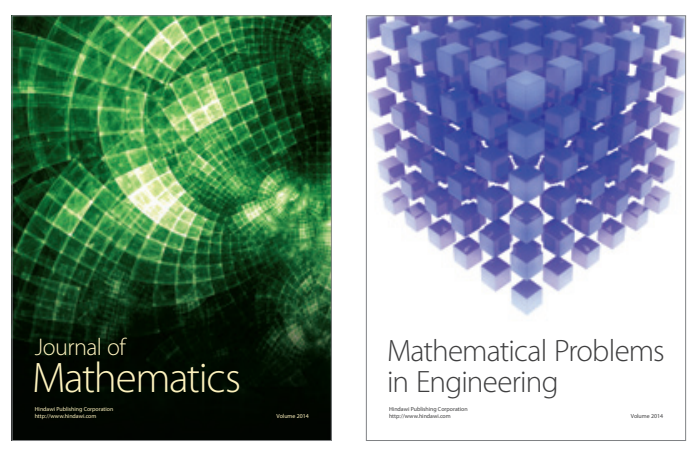

Mathematical Problems in Engineering
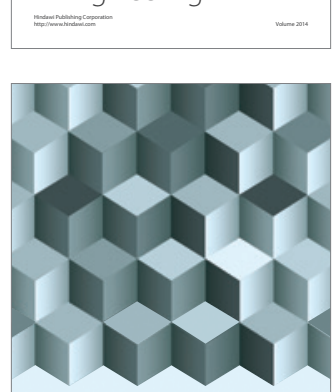

Journal of

Function Spaces
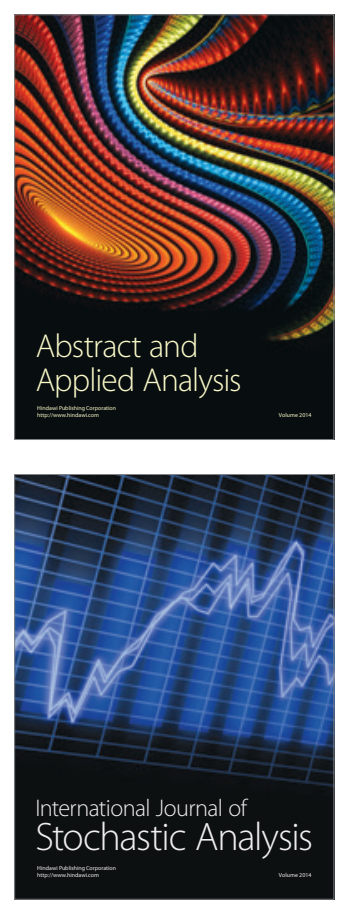

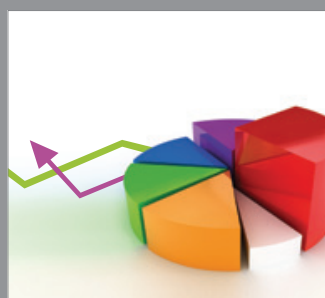

ournal of

Probability and Statistics

Promensencen
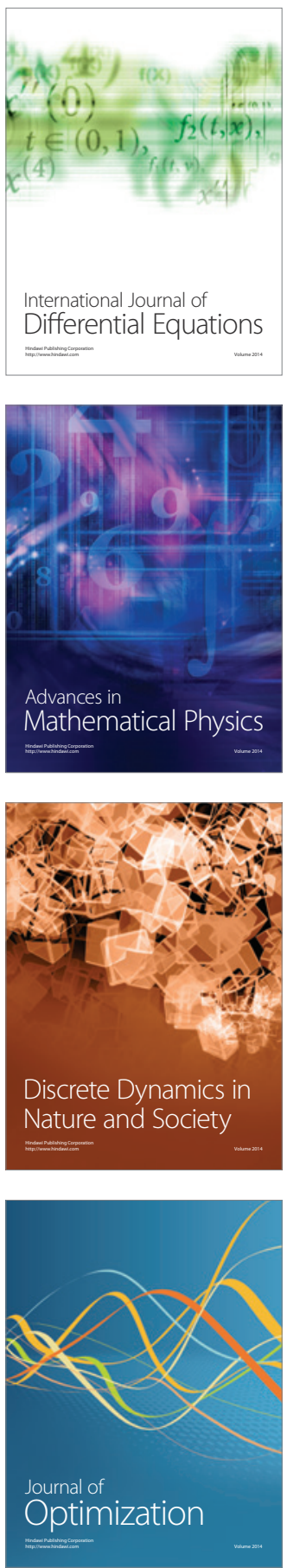